\title{
ON THE FIRST-PASSAGE TIME OF INTEGRATED BROWNIAN MOTION
}

\author{
CHRISTIAN H. HESSE
}

Received 30 January 2004 and in revised form 29 September 2004

Let $\left(B_{t} ; t \geq 0\right)$ be a Brownian motion process starting from $B_{0}=v$ and define $X_{\nu}(t)=$ $\int_{0}^{t} B_{s} d s$. For $a \geq 0$, set $\tau_{a, v}:=\inf \left\{t: X_{v}(t)=a\right\}$ (with inf $\phi=\infty$ ). We study the conditional moments of $\tau_{a, v}$ given $\tau_{a, v}<\infty$. Using martingale methods, stopping-time arguments, as well as the method of dominant balance, we obtain, in particular, an asymptotic expansion for the conditional mean $E\left(\tau_{a, v} \mid \tau_{a, v}<\infty\right)$ as $\nu \rightarrow \infty$. Through a series of simulations, it is shown that a truncation of this expansion after the first few terms provides an accurate approximation to the unknown true conditional mean even for small $\nu$.

\section{Introduction}

First-passage time problems (FPTPs) for integrated Markov processes constitute a set of old and challenging problems of probability. They arise both in theoretical and in applied contexts. Our motivation to study them stems from previous work on a stochastic model for particle sedimentation in fluids where one such problem arises naturally; see Hesse and Tory [4]. In stochastic approaches to sedimentation, particle velocity is often modeled as Brownian motion $\left(B_{t} ; t \geq 0\right)$. Particle position then is the integral over this Brownian motion and the question when a particle first travels through a specified distance then leads to the FPTP for this integrated Markov process.

FPTPs for integrated Markov processes are complicated by the fact that the integral of a Markov process is no longer Markovian. However, the two-dimensional process such as

$$
\left(B_{t}, \int_{0}^{t} B_{s} d s ; t \geq 0\right)
$$

in our case is Markovian. The process in (1.1) is often called the Kolmogorov diffusion since its study was initiated by Kolmogorov [5].

This lifting of the original process into two dimensions so as not to lose the Markovian property often necessitates additional boundary and initial conditions which are frequently not available. Specifically, for our FPTP of integrated Brownian motion, we circumvent this impasse by considering a slightly perturbed process, hence introducing a boundary with a specification for the Laplace transform of the first-passage time density 
on this boundary and later letting the boundary go to infinity in a controlled fashion. This is demonstrated in Sections 2 and 3. In Section 2, using martingale methods and stopping-time arguments, a system of partial differential equations for the conditional moments of the FPT distribution of integrated Brownian motion is derived. Here and below "conditional" is meant to indicate "given that the first-passage time is finite." In Section 3, an asymptotic expansion for the conditional mean first-passage time is derived. Section 4 reports on the results of a series of simulations which show that the truncated asymptotic expansion for the conditional mean turns out to be an accurate approximation to the unknown conditional mean.

We now define our problem rigorously and mention related works. Let $\left(B_{t} ; t \geq 0\right)$ be a Brownian motion process with diffusion parameter $\sigma^{2}$ starting from $E\left(B_{t}\right)=B_{0}=v$ and define the position process $\left(X_{v}(t) ; t \geq 0\right)$ through

$$
X_{v}(t)=X_{v}(0)+\int_{0}^{t} B_{s} d s
$$

Then

$$
\tau_{a, v}= \begin{cases}\inf \left\{t: X_{\nu}(t)=X_{\nu}(0)+a\right\} & \text { if this set is not empty } \\ \infty & \text { otherwise }\end{cases}
$$

is the first-passage time of the position process to the state at distance $a$. We are interested in the conditional moments

$$
E\left(\tau_{a, v} \mid \tau_{a, v}<\infty\right)
$$

as functions of $a, v$, and $\sigma^{2}$.

Early studies of integrated Brownian motion are McKean [10], Goldman [2], and Gor'kov [3]. McKean investigated joint probability densities of certain first-passage times $\tau$ and corresponding hitting velocities $B_{\tau}$. Goldman [2] has derived an expression of the joint probability density function of the so-called half-winding time $\tau^{\prime}$ of the process and the velocity $B_{\tau^{\prime}}$. In Gor'kov [3], a formula for the density of $B_{\tau^{*}}$, where $\tau^{*}=\min \{t$ : $\left.X_{v}(t)=b, B_{t} \geq 0\right\}, b>0$, is computed.

More recently, Lefebvre [8] has obtained joint moment-generating functions related to certain first-passage times as well as-in a special case-the probability density function of the first hitting place. His method is based on solving the appropriate Kolmogorov backward equations. Lachal [6] presents an elementary procedure for rederiving some of Lefebvre [8] formulas as well as for deriving explicitly the probability laws of certain firstpassage times. Also, Lachal [7] contains results on first-passage times which are obtained by martingale methods. Finally, Lefebvre [9] gives results on certain first-passage problems for degenerate two-dimensional diffusion processes. All of these approaches lead to rather complicated integral expressions which one does not seem to be able to evaluate analytically to obtain moment information. 


\section{A system of equations for the conditional moments}

In this section, we derive a system of partial differential equations for the moments of the conditional first-passage time $\tau_{a, \nu}$. Let $(\Omega, \mathscr{F}, P)$ be a probability space on which all upcoming processes will be defined.

Consider the process

$$
\left(X_{\nu}(s), B_{s} ; s \geq 0\right), \quad X_{\nu}(0)=-x \leq 0, B_{0}=\nu,
$$

with an absorbing boundary at the plane $(0, \mathbb{R})$ and let $p\left(x_{t}, v_{t}, t \mid x, \nu\right)$ be the probability density associated with

$$
X_{v}(0)=-x, \quad B_{0}=v, \quad X_{v}(t)=x_{t}, \quad B_{t}=v_{t}, \quad X_{v}(s)<0, \quad \forall s \in[0, t) .
$$

The density $p$ depends on the starting distance to the boundary, of course. Also, let

$$
P(y, t \mid x, v)=\int_{-\infty}^{y} \int_{-\infty}^{+\infty} p\left(x_{t}, v_{t}, t \mid x, v\right) d v_{t} d x_{t}
$$

so that $P(0, t \mid x, \nu)$ is the probability that the boundary has not been reached prior to time $t$. We also introduce the Laplace transform of the FPT distribution, namely,

$$
\Psi_{s}(x, v):=\int_{0}^{\infty} e^{-s t} \frac{\partial}{\partial t}(1-P(0, t \mid x, v)) d t
$$

A key instrument in our derivation will be the random Laplace transform $\Psi_{s}\left(-X_{v}(t), B_{t}\right)$. To state our results, we also introduce the notation

$$
\begin{aligned}
\partial_{1} \Psi_{s}(y, z) & =\frac{\partial}{\partial y} \Psi_{s}(y, z), \\
\partial_{2} \Psi_{s}(y, z) & =\frac{\partial}{\partial z} \Psi_{s}(y, z), \\
\partial_{22} \Psi_{s}(y, z) & =\frac{\partial^{2}}{\partial z^{2}} \Psi_{s}(y, z),
\end{aligned}
$$

An important role in obtaining conditional moments of the FPT distribution will be played by the process

$$
\left(Z(t):=\exp (-s t) \Psi_{s}\left(-X_{\nu}(t), B_{t}\right) ; 0 \leq t \leq \tau_{x, \nu}\right),
$$

which is defined for all fixed positive $s$.

Theorem 2.1. The stochastic Ito-differential $d Z(t)$ of $Z(t)$ is given by

$$
\begin{aligned}
d Z(t)= & \exp (-s t) \partial_{2} \Psi_{s}\left(-x_{t}, v_{t}\right) \sigma d W_{t} \\
& +\exp (-s t)\left[\frac{\sigma^{2}}{2} \partial_{22} \Psi_{s}\left(-x_{t}, v_{t}\right)-B_{t} \partial_{1} \Psi_{s}\left(-x_{t}, v_{t}\right)-s \Psi_{s}\left(-x_{t}, v_{t}\right)\right] d t
\end{aligned}
$$

where $W_{t}$ is standard Brownian motion. 
Proof. We compute explicitly the increment of $Z(t)$ over a time interval of length $\triangle t$. It is

$$
\begin{aligned}
\Delta Z:= & Z(t+\Delta t)-Z(t) \\
= & {[\exp (-s(t+\Delta t))-\exp (-s t)]\left[\Psi_{s}\left(-x_{t+\Delta t}, v_{t+\Delta t}\right)-\Psi_{s}\left(-x_{t}, v_{t}\right)\right] } \\
& +[\exp (-s(t+\Delta t))-\exp (-s t)] \Psi_{s}\left(-x_{t}, v_{t}\right) \\
& +\exp (-s t)\left[\Psi_{s}\left(-x_{t+\Delta t}, v_{t+\Delta t}\right)-\Psi_{s}\left(-x_{t}, v_{t}\right)\right]
\end{aligned}
$$

Retaining terms of order $\Delta t$ only, this becomes

$$
\begin{aligned}
\Delta Z=-s \exp (-s t) & \Psi_{s}\left(-x_{t}, v_{t}\right) \Delta t \\
+\exp (-s t)[ & -\partial_{1} \Psi_{s}\left(-x_{t}, v_{t}\right) B_{t} \Delta t+\partial_{2} \Psi_{s}\left(-x_{t}, v_{t}\right) \Delta B_{t} \\
& \left.+\frac{1}{2} \partial_{22} \Psi_{s}\left(-x_{t}, v_{t}\right)\left(\Delta B_{t}\right)^{2}\right]+o\left((\Delta t)^{3 / 2}\right) .
\end{aligned}
$$

Noting that $\Delta B_{t}=\sigma \Delta W_{t},\left(\Delta B_{t}\right)^{2}=\sigma^{2}\left(\Delta W_{t}\right)^{2}=\sigma^{2} \Delta t$, and including terms such as $\Delta t \Delta W_{t}$ and higher-order terms in the $o\left((\Delta t)^{3 / 2}\right)$ summand, we arrive at

$$
\begin{aligned}
\Delta Z= & \exp (-s t) \partial_{2} \Psi_{s}\left(-x_{t}, v_{t}\right) \sigma \Delta W_{t} \\
& +\exp (-s t)\left[\frac{\sigma^{2}}{2} \partial_{22} \Psi_{s}\left(-x_{t}, v_{t}\right)-B_{t} \partial_{1} \Psi_{s}\left(-x_{t}, v_{t}\right)-s \Psi_{s}\left(-x_{t}, v_{t}\right)\right] \Delta t+\sigma\left((\Delta t)^{3 / 2}\right)
\end{aligned}
$$

The result then follows by replacing infinitesimal increments by differentials.

Theorem 2.2. The process $\left(Z(t) ; 0 \leq t \leq \tau_{x, v}\right)$ as defined before Theorem 2.1 is a local martingale relative to the family of $\sigma$-algebras generated by $(Z(s) ; s \leq t)$.

Proof. The proof is based on stopping-time arguments and is simple. The details are omitted.

From Theorem 2.1, we can deduce the representation

$$
\begin{aligned}
Z(t)= & \Psi_{s}(x, \nu)+\int_{0}^{t} \exp (-s \tau) \partial_{2} \Psi_{s} \sigma d W_{\tau} \\
& +\int_{0}^{t} \exp (-s \tau)\left[\frac{\sigma^{2}}{2} \partial_{22} \Psi_{s}-B_{t} \partial_{1} \Psi_{s}-s \Psi_{s}\right] d \tau .
\end{aligned}
$$

The local martingale property of $Z(t)$ implies that the third summand on the right side of (2.11) must vanish for all $t \geq 0$. From this, we may deduce that

$$
\frac{\sigma^{2}}{2} \frac{\partial^{2} \Psi_{s}(x, \nu)}{\partial \nu^{2}}-v \frac{\partial \Psi_{s}(x, \nu)}{\partial x}-s \Psi_{s}(x, \nu)=0 .
$$

The absorbing boundary condition gives

$$
\Psi_{s}(0, v)=1, \quad \forall s>0, \forall v>0 .
$$


As defined, the function $\Psi_{s}$ possesses the representation

$$
\Psi_{s}(x, v)=\sum_{n=0}^{\infty} \frac{(-s)^{n}}{n !} m_{n}(x, \nu),
$$

where $m_{n}(x, v)$ is the $n$th conditional moment of the FPT distribution, that is, $E\left(\tau_{x, v}^{n}\right.$ । $\left.\tau_{x, \nu}<\infty\right)$.

Substituting (2.14) into (2.12) and equating coefficients of powers of $s$, we arrive at the following system of partial differential equations for $m_{n}$ :

$$
\begin{gathered}
\frac{\sigma^{2}}{2} \frac{\partial^{2} m_{n}(x, v)}{\partial v^{2}}-v \cdot \frac{\partial m_{n}(x, v)}{\partial x}=-n \cdot m_{n-1}(x, v) \\
m_{n}(0, v)=0, \quad \forall n \geq 1, v>0 \\
m_{0}(0, v)=1 .
\end{gathered}
$$

For the conditional mean FPT, we get, suppressing the subscripts of $m$,

$$
\begin{gathered}
\frac{\sigma^{2}}{2} \frac{\partial^{2} m(x, v)}{\partial \nu^{2}}-v \cdot \frac{\partial m(x, v)}{\partial x}=-1, \\
m(0, v)=0, \quad \forall v>0 .
\end{gathered}
$$

A difficulty with the systems (2.15), (2.16) and (2.17), (2.18) arises from the lack of sufficient boundary or initial information. Although (2.16) is obvious since

$$
\lim _{x \rightarrow 0+} \lim _{\nu \rightarrow \nu^{*}} m_{n}(x, \nu)=m_{n}\left(0, v^{*}\right), \quad \forall v^{*}>0, \forall n \geq 1
$$

it is unclear how this limit behaves for $v^{*}<0$. Hence, the boundary value problem is not well-posed. On the other hand, to find $\lim _{x \rightarrow x^{*}} \lim _{\nu \rightarrow v^{*}} m_{n}(x, v)$ for a given $v^{*}$ and all $x^{*} \geq 0$ is as difficult as the original FPTP itself. Therefore, also the initial boundary value problem is ill-posed and it seems that we have arrived at an impasse. In the following section, we demonstrate how to circumvent this difficulty and derive an asymptotic expansion for $m(x, \nu)$ as $\nu \rightarrow \infty$.

\section{An asymptotic expansion for the mean}

A way out of the dilemma indicated at the end of Section 2 is based on the following modified process. For some $v_{0}>v$ define

$$
\tilde{B}_{t}:= \begin{cases}B_{t} & \text { if } B_{s}<v_{0}, \quad \forall s \leq t \\ \nu_{0}, & \forall t \geq \tau_{0}:=\min \left\{t: B_{t}=v_{0}\right\}\end{cases}
$$

and let $\tilde{X}_{v}(t)=X_{v}(0)+\int_{0}^{t} \tilde{B}_{s} d s$. The process $\left(\tilde{X}_{v}(t), \tilde{B}_{t} ; t \geq 0\right)$ has the same sample paths as $\left(X_{\nu}(t), B_{t} ; t \geq 0\right)$ except when the Brownian motion process hits $\nu_{0}$. When this happens, the Brownian motion terminates a.s. at $v_{0}$. Also, let

$$
\tau_{a, \nu, \nu_{0}}:= \begin{cases}\min \left\{t \geq 0: \tilde{X}_{\nu}(t)=X_{\nu}(0)+a\right\} & \text { if this set is not empty, } \\ \infty & \text { if } \tilde{X}_{\nu}(t) \neq X_{\nu}(0)+a, \quad \forall t \geq 0,\end{cases}
$$


be the FPT of the modified process on $X_{v}(0)+a$ and

$$
\tilde{m}_{n}(x, \nu):=E\left(\tau_{x, \nu, \nu_{0}}^{n} \mid \tau_{x, \nu, \nu_{0}}<\infty\right) .
$$

Then the $\tilde{m}_{n}(x, v)$ also satisfy $(2.15),(2.16)$, as well as

$$
\tilde{m}_{n}\left(x, v_{0}\right)=\left(\frac{x}{v_{0}}\right)^{n}, \quad \forall x \geq 0, \forall n \geq 1
$$

Furthermore, both $\tau_{x, \nu}$ and $\tau_{x, \nu_{0}, \nu_{0}}$ are stopping times with respect to the family of $\sigma$ algebras generated by $\left(X_{v}(t), B_{t} ; t \geq 0\right)$ and we have $\tau_{x, \nu, \nu_{0}} \stackrel{\nu_{0} \rightarrow \infty}{\longrightarrow} \tau_{x, \nu}$ a.s. From these considerations, the following approach is suggested for the mean FPT. For (2.17), the initial boundary value problem is solved with $(2.18)$ and

$$
m\left(x, v_{0}\right)=\frac{x}{v_{0}}, \quad \forall x \geq 0, v_{0} \text { large. }
$$

Then the limit as $\nu_{0} \rightarrow \infty$ is taken. Writing

$$
\hat{m}(c, v)=\int_{0}^{\infty} \exp (-c x) m(x, v) d x,
$$

one obtains

$$
\begin{gathered}
\frac{\sigma^{2}}{2} \frac{d^{2} \hat{m}(c, v)}{d \nu^{2}}-c \nu \hat{m}(c, \nu)=-c^{-1}, \\
\hat{m}\left(c, v_{o}\right)=v_{0}^{-1} c^{-2} .
\end{gathered}
$$

The substitution

$$
\begin{gathered}
\omega=2^{1 / 3} c^{1 / 3} \sigma^{-2 / 3} v \\
\omega_{0}=2^{1 / 3} c^{1 / 3} \sigma^{-2 / 3} v_{0}
\end{gathered}
$$

transforms (3.7) and (3.8) into

$$
\begin{gathered}
\frac{d^{2} \hat{m}(c, \omega)}{d \omega^{2}}-\omega \cdot \hat{m}(c, \omega)=-2^{1 / 3} \sigma^{-2 / 3} c^{-5 / 3}, \\
\hat{m}\left(c, \omega_{0}\right)=2^{1 / 3} \sigma^{-2 / 3} c^{-5 / 3} \omega_{0}^{-1},
\end{gathered}
$$

whose homogeneous part is a one-dimensional Schrödinger equation. We now use methods of global analysis such as WKB analysis and the method of dominant balance on this Schrödinger equation (see, e.g., Bender and Orszag [1]). If the power series $\sum_{n=0}^{\infty} p(n) \omega^{n}$ is substituted for $\hat{m}$ into (3.10), the coefficients are seen to satisfy

$$
\begin{gathered}
p(3 n+2)=0, \\
p(3 n+1)=\frac{p(1) \cdot \Gamma(4 / 3)}{9^{n} n ! \Gamma(n+4 / 3)}, \\
p(3 n)=\frac{p(0) \cdot \Gamma(2 / 3)}{9^{n} n ! \Gamma(n+2 / 3)}, \quad \forall n \geq 0 .
\end{gathered}
$$


Performing a variation of parameters and writing

$$
\begin{aligned}
& A(\omega):=3^{-2 / 3} \sum_{n=0}^{\infty} \frac{\omega^{3 n}}{9^{n} n ! \Gamma(n+2 / 3)}-3^{-4 / 3} \sum_{n=0}^{\infty} \frac{\omega^{3 n+1}}{9^{n} n ! \Gamma(n+4 / 3)}, \\
& B(\omega):=3^{-1 / 6} \sum_{n=0}^{\infty} \frac{\omega^{3 n}}{9^{n} n ! \Gamma(n+2 / 3)}+3^{-5 / 6} \sum_{n=0}^{\infty} \frac{\omega^{3 n+1}}{9^{n} n ! \Gamma(n+4 / 3)},
\end{aligned}
$$

this leads to

$$
\hat{m}(c, \omega)=\pi \cdot 2^{1 / 3} \sigma^{-2 / 3} c^{-5 / 3}\left[B(\omega) \int_{\omega}^{\infty} A(t) d t+A(\omega) \int_{0}^{\omega} B(t) d t\right]+k \cdot A(\omega),
$$

where $k=k(c)$ is a constant that needs to be fitted so that (3.14) satisfies the initial condition (3.11).

$A(\omega)$ and $B(\omega)$ in (3.14) are the so-called Airy functions and it is well known that $A(\omega)$ decays exponentially as $\omega \rightarrow \infty$. In fact

$$
A(\omega) \sim 2^{-1} \pi^{-1 / 2} \omega^{-1 / 4} \exp \left(-\frac{2}{3} \omega^{3 / 2}\right), \quad \text { as } \omega \rightarrow \infty .
$$

Hence it can be expected that the asymptotic expansion of (3.14) as $\omega \rightarrow \infty$ equals the asymptotic expansion of merely the first two summands of (3.14). We confirm this with the following argument. The strategy is to first peel off the leading asymptotic behavior, then, after having removed this, to determine the leading behavior of the remainder, and so forth. The first step in this procedure is to assume

$$
\frac{d^{2} \hat{m}(c, \omega)}{d \omega^{2}} \sim 0, \quad \text { as } \omega \rightarrow \infty,
$$

which yields

$$
\hat{m}(c, \omega) \sim 2^{1 / 3} \sigma^{-2 / 3} c^{-5 / 3} \omega^{-1}, \quad \text { as } \omega \rightarrow \infty .
$$

Corrections to this leading term are obtained by writing

$$
\hat{m}(c, \omega)=2^{1 / 3} \sigma^{-2 / 3} c^{-5 / 3}\left(\omega^{-1}+\varepsilon(\omega)\right), \quad \text { as } \omega \rightarrow \infty,
$$

where the correction term is of smaller order than $\omega^{-1}$. It is easily determined that $\varepsilon(\omega)$ satisfies the differential equation

$$
\frac{d^{2} \varepsilon(\omega)}{d \omega^{2}}+\frac{2}{\omega^{3}}=\omega \cdot \varepsilon(\omega)
$$

Again, setting $d^{2} \varepsilon(\omega) / d \omega^{2} \sim 0$ (as $\left.\omega \rightarrow \infty\right)$, we see that $\varepsilon(\omega) \sim 2 \omega^{-4}$ and continuing with this method, one obtains the full asymptotic power series expansion

$$
\hat{m}(c, \omega) \sim 2^{1 / 3} \sigma^{-2 / 3} c^{-5 / 3} \sum_{n=0}^{\infty} \frac{(3 n) !}{3^{n} m !} \omega^{-3 n-1}
$$


This indeed is the asymptotic expansion of the first two summands on the right-hand side of (3.14).

From (3.20), we arrive at

$$
m(x, \nu) \sim \sum_{n=0}^{\infty} \frac{(3 n) ! \sigma^{2 n} x^{n+1}}{n !(n+1) ! 6^{n} \nu^{3 n+1}} .
$$

It is easily checked by differentiating termwise that the asymptotic expansion in (3.21) formally satisfies (2.17) and the boundary condition (2.18). However, the sum in (3.21) does not converge for any nonzero value of $\sigma^{2} x \nu^{-3}$. Although this might be surprising at first, it is well known that most problems in perturbation theory or WKB analysis lead to divergent series. It was Poincaré who introduced the concept of divergent asymptotic expansions into mathematics and demonstrated that formal solutions of differential equations are asymptotic expansions of actual solutions. In fact, typically, optimally truncated divergent series constitute accurate approximations to the actual solutions, see again Bender and Orszag [1]. We truncate (3.21) after the third summand and use

$$
\bar{m}(x, v)=\frac{x}{v}+\frac{\sigma^{2}}{2} \frac{x^{2}}{v^{4}}+\frac{5 \sigma^{4}}{3} \frac{x^{3}}{v^{7}}
$$

as our approximation.

\section{Simulations}

We performed an extensive series of simulations to evaluate the quality of the approximation (3.22) for various distances $x$ to boundary, initial velocities $v$, and diffusion parameters $\sigma$. The simulations indicate that the approximation is accurate whenever $\sigma^{2} x v^{-3}<1 / 4$.

Summaries of the simulations are reported in Tables 4.1, 4.2, and 4.3. The following notation is used (for convenience, the dependence on $x, \nu, \sigma$ was dropped):

$$
A_{1}=\frac{x}{v}, \quad A_{2}=\frac{\sigma^{2}}{2} \frac{x^{2}}{v^{4}}, \quad A_{3}=\frac{5 \sigma^{4}}{3} \frac{x^{3}}{v^{7}}
$$

$m_{s}=$ sample averages of simulated first-passage times for given $x, \nu, \sigma^{2}$,

$$
\begin{gathered}
M_{1}=10^{3} \cdot \frac{m_{s}-A_{1}}{A_{1}}, \quad M_{2}=10^{3} \cdot \frac{m_{s}-\left(A_{1}+A_{2}\right)}{A_{1}}, \quad M_{3}=10^{3} \cdot \frac{m_{s}-\left(A_{1}+A_{2}+A_{3}\right)}{A_{1}}, \\
S D\left(M_{i}\right)=\text { sample standard deviation of } M_{i}, \quad i=1,2,3, \\
t_{i}=\frac{M_{i}}{S D\left(M_{i}\right)} .
\end{gathered}
$$

At time $t=0$, a total of 2000 particles were started with initial velocity $\nu$ (for $\nu=$ $2,4,6,8$ ) and diffusion parameter $\sigma$ (for $\sigma=0.1,0.3,0.7$ ). We recorded when these particles first crossed boundaries at distances $x=2,4,8,12,20$ and computed $m_{s}, M_{i}, S D\left(M_{i}\right)$, and $t_{i}$.

The values of $t_{1}$ indicate that the first-order approximation $A_{1}$ tends to underestimate the mean first-passage time. On the other hand, with $\left|t_{3}\right| \leq 2$ in almost all cases 
Table 4.1. $M_{i}, t_{i}$ for various values of $x, v$ and $\sigma=0.1$.

\begin{tabular}{|c|c|c|c|c|c|c|c|c|c|c|c|c|}
\hline \multicolumn{7}{|c|}{$\nu=2$} & \multicolumn{6}{|c|}{$\nu=4$} \\
\hline$x$ & $M_{1}$ & $M_{2}$ & $M_{3}$ & $t_{1}$ & $t_{2}$ & $t_{3}$ & $M_{1}$ & $M_{2}$ & $M_{3}$ & $t_{1}$ & $t_{2}$ & $t_{3}$ \\
\hline 2 & 6.1 & -6.41 & -6.51 & 0.9 & -1.00 & -1.02 & -0.6 & -2.12 & -2.12 & -0.2 & -0.95 & -0.95 \\
\hline 4 & 9.4 & -15.59 & -16.01 & 1.0 & -1.71 & -1.75 & 2.1 & -1.07 & -1.08 & 0.6 & -0.33 & -0.33 \\
\hline 8 & 26.0 & -24.02 & -25.69 & 1.9 & -1.84 & -1.97 & 8.3 & 2.09 & 2.06 & 1.8 & 0.45 & 0.45 \\
\hline 12 & 54.9 & -20.13 & -23.88 & 3.3 & -1.22 & -1.4 & 12.9 & 3.49 & 3.43 & 2.2 & 0.61 & 0.60 \\
\hline 20 & 10.0 & -14.97 & -25.38 & 5.0 & -0.68 & -1.15 & 19.4 & 3.78 & 3.62 & 2.6 & 0.51 & 0.49 \\
\hline \multicolumn{7}{|c|}{$v=6$} & \multicolumn{6}{|c|}{$v=8$} \\
\hline$x$ & $M_{1}$ & $M_{2}$ & $M_{3}$ & $t_{1}$ & $t_{2}$ & $t_{3}$ & $M_{1}$ & $M_{2}$ & $M_{3}$ & $t_{1}$ & $t_{2}$ & $t_{3}$ \\
\hline 2 & 0.7 & 0.22 & 0.22 & 0.5 & 0.18 & 0.18 & 0.8 & 0.65 & 0.65 & & 0.90 & 0.90 \\
\hline 4 & 0.1 & -0.86 & -0.86 & 0.0 & -0.49 & -0.49 & 1.5 & 1.15 & 1.15 & 1.3 & 1.03 & 1.03 \\
\hline 8 & -0.4 & -2.23 & -2.23 & -0.1 & -0.89 & -0.89 & 1.3 & 0.53 & 0.53 & 0.8 & 0.33 & 0.33 \\
\hline 12 & -0.1 & -2.91 & -2.92 & -0.0 & -0.94 & -0.95 & 1.2 & -0.01 & -0.01 & 0.6 & 0.00 & 0.00 \\
\hline 20 & 2.2 & -2.39 & -2.41 & 0.5 & -0.60 & -0.60 & 1.9 & -0.03 & -0.03 & 0.7 & -0.01 & -0.01 \\
\hline
\end{tabular}

Table 4.2. $M_{i}, t_{i}$ for various values of $x, v$ and $\sigma=0.3$.

\begin{tabular}{|c|c|c|c|c|c|c|c|c|c|c|c|c|}
\hline \multicolumn{7}{|c|}{$v=2$} & \multicolumn{6}{|c|}{$v=4$} \\
\hline$x$ & $M_{1}$ & $M_{2}$ & $M_{3}$ & $t_{1}$ & $t_{2}$ & $t_{3}$ & $M_{1}$ & $M_{2}$ & $M_{3}$ & $t_{1}$ & $t_{2}$ & $t_{3}$ \\
\hline 2 & 81.0 & -31.52 & -39.96 & 3.8 & -1.49 & -1.89 & 16.5 & 2.44 & 2.31 & 2.3 & 0.35 & 0.33 \\
\hline 4 & 238.4 & 13.44 & -20.31 & 6.3 & 0.36 & -0.54 & 26.5 & -1.60 & -2.13 & 2.7 & -0.16 & -0.22 \\
\hline 8 & 846.9 & 396.91 & 261.91 & 6.1 & 2.85 & 1.88 & 43.3 & -12.92 & -15.02 & 3.1 & -0.91 & -1.06 \\
\hline 12 & 601.0 & 926.05 & 622.29 & 4.3 & 2.50 & & 67.9 & -16.46 & -21.21 & 3.8 & -0.93 & -1.20 \\
\hline 20 & 3063.4 & 938.43 & 1094.68 & 4.0 & 2.53 & 1.43 & 33.9 & -6.69 & -19.87 & 5.6 & -0.28 & -0.83 \\
\hline \multicolumn{7}{|c|}{$v=6$} & \multicolumn{6}{|c|}{$\nu=8$} \\
\hline$x$ & $M_{1}$ & $M_{2}$ & $M_{3}$ & $t_{1}$ & $t_{2}$ & $t_{3}$ & $M_{1}$ & $M_{2}$ & $M_{3}$ & $t_{1}$ & $t_{2}$ & $t_{3}$ \\
\hline 2 & 10.1 & 5.90 & 5.89 & 2.7 & 1.57 & 1.57 & 0.1 & -1.68 & -1.68 & 0.0 & -0.70 & -0.70 \\
\hline 4 & 16.4 & 8.07 & 8.02 & 3.1 & 1.53 & 1.52 & -1.3 & -4.85 & -4.86 & -0.4 & -1.43 & -1.43 \\
\hline 8 & 25.8 & 9.15 & 8.96 & 3.4 & 1.22 & 1.19 & 0.9 & -6.11 & -6.14 & 0.2 & -1.28 & -1.28 \\
\hline 12 & 31.9 & 6.87 & 6.45 & 3.5 & 0.74 & 0.70 & 3.4 & -7.13 & -7.21 & 0.6 & -1.22 & -1.24 \\
\hline 20 & 52.4 & 10.76 & 9.60 & 4.4 & 0.90 & 0.80 & 10.2 & -7.37 & -7.58 & 1.4 & -0.99 & -1.01 \\
\hline
\end{tabular}

Table 4.3. $M_{i}, t_{i}$ for various values of $x, v$ and $\sigma=0.7$.

\begin{tabular}{|c|c|c|c|c|c|c|c|c|c|c|c|c|}
\hline \multicolumn{7}{|c|}{$v=4$} & \multicolumn{6}{|c|}{$v=6$} \\
\hline$x$ & $M_{1}$ & $M_{2}$ & $M_{3}$ & $t_{1}$ & $t_{2}$ & $t_{3}$ & $M_{1}$ & $M_{2}$ & $M_{3}$ & $t_{1}$ & $t_{2}$ & $t_{3}$ \\
\hline 2 & 52.8 & -23.76 & -27.61 & 3.1 & -1.41 & -1.64 & 13.6 & -9.11 & -9.45 & 1.5 & -1.04 & -1.08 \\
\hline 4 & 107.6 & -45.49 & -61.13 & 4.4 & -1.87 & -2.51 & 34.6 & -10.75 & -12.12 & 2.7 & -0.86 & -0.97 \\
\hline 8 & 213.6 & -92.68 & -155.21 & 6.0 & -2.61 & -4.36 & 88.8 & -1.96 & -7.45 & 4.9 & -0.11 & -0.41 \\
\hline 12 & 300.4 & -158.96 & -299.64 & 6.8 & -3.63 & -6.85 & 138.4 & 2.28 & -10.07 & 6.0 & 0.10 & -0.44 \\
\hline 20 & 3195.5 & 2429.93 & 2039.14 & 1.1 & 0.87 & 0.74 & 262.7 & 35.90 & 1.59 & 7.5 & 1.04 & 0.05 \\
\hline \multicolumn{7}{|c|}{$v=8$} & \multicolumn{6}{|c|}{$v=10$} \\
\hline$x$ & $M_{1}$ & $M_{2}$ & $M_{3}$ & $t_{1}$ & $t_{2}$ & $t_{3}$ & $M_{1}$ & $M_{2}$ & $M_{3}$ & $t_{1}$ & $t_{2}$ & $t_{3}$ \\
\hline 2 & -4.4 & -13.97 & -14.03 & -0.8 & -2.52 & -2.53 & 10.4 & 3.66 & 3.63 & 2.1 & 0.76 & 0.75 \\
\hline 4 & -7.0 & -26.18 & -26.42 & -0.8 & -3.25 & -3 & 18.0 & 4.52 & 4.40 & 2.6 & 0.66 & 0.65 \\
\hline 8 & 4.4 & -33.89 & -34.87 & 0.3 & -2.93 & -3.0 & 39.3 & 12.40 & 11.92 & 4.0 & 1.27 & 1.22 \\
\hline 12 & 23.9 & -33.54 & -35.74 & 1.6 & -2.33 & -2.49 & 55.4 & 15.03 & 13.95 & 4.5 & 1.23 & 1.15 \\
\hline 20 & 64.0 & -31.74 & -37.84 & 3.4 & -1.69 & -2.01 & 80.8 & 13.56 & 10.55 & 5.1 & 0.86 & 0.63 \\
\hline
\end{tabular}




\section{On the first-passage time of integrated Brownian motion}

considered, the third-order approximation $A_{1}+A_{2}+A_{3}$ seems to approximate the mean first-passage time very well. The difference between the second-order and the third-order approximation is rather small in most cases.

\section{References}

[1] C. M. Bender and S. A. Orszag, Advanced Mathematical Methods for Scientists and Engineers, International Series in Pure and Applied Mathematics, McGraw-Hill Book, New York, 1978.

[2] M. Goldman, On the first passage of the integrated Wiener process, Ann. Math. Statist. 42 (1971), $2150-2155$.

[3] J. P. Gor'kov, A formula for the solution of a certain boundary value problem for the stationary equation of Brownian motion, Dokl. Akad. Nauk SSSR 223 (1975), no. 3, 525-528 (Russian).

[4] C. H. Hesse and E. M. Tory, The stochastics of sedimentation, Adv. Fluid Mech. 7 (1996), 199240.

[5] A. Kolmogorov, Zufällige Bewegungen (zur Theorie der Brownschen Bewegung), Ann. of Math. (2) 35 (1934), no. 1, 116-117 (German).

[6] A. Lachal, L'intégrale du mouvement brownien, J. Appl. Probab. 30 (1993), no. 1, 17-27 (French).

[7] Some martingales related to the integral of Brownian motion. Applications to the passage times and transience, J. Theoret. Probab. 11 (1998), no. 1, 127-156.

[8] M. Lefebvre, First-passage densities of a two-dimensional process, SIAM J. Appl. Math. 49 (1989), no. $5,1514-1523$.

[9] _ First-passage problems for degenerate two-dimensional diffusion processes, Test 12 (2003), no. 1, 125-139.

[10] H. P. McKean Jr., A winding problem for a resonator driven by a white noise, J. Math. Kyoto Univ. 2 (1963), 227-235.

[11] E. M. Tory and C. H. Hesse, Theoretical and experimental evidence for a Markov model for sedimentation, Adv. Fluid Mech. 7 (1996), 241-280.

Christian H. Hesse: Institut für Stochastik und Anwendungen, Fachbereich Mathematik, Universität Stuttgart, 70569 Stuttgart, Germany

E-mail address: hesse@mathematik.uni-stuttgart.de 


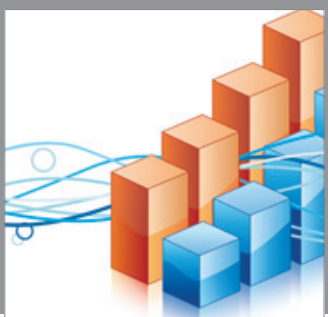

Advances in

Operations Research

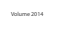

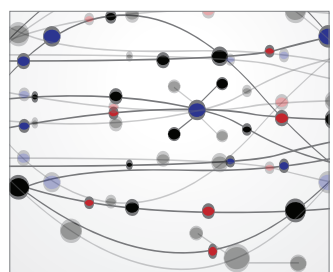

\section{The Scientific} World Journal
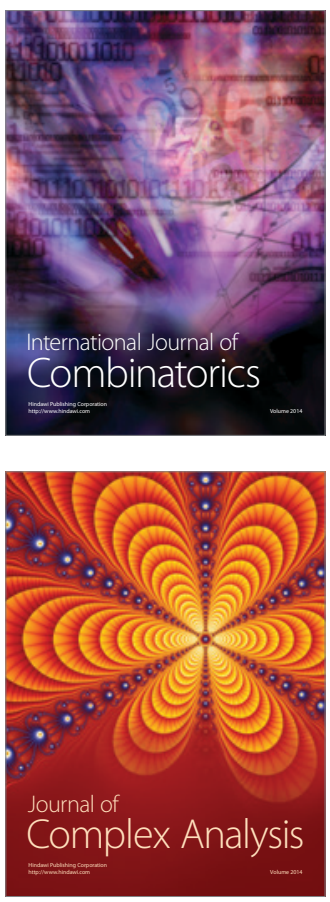

International Journal of

Mathematics and

Mathematical

Sciences
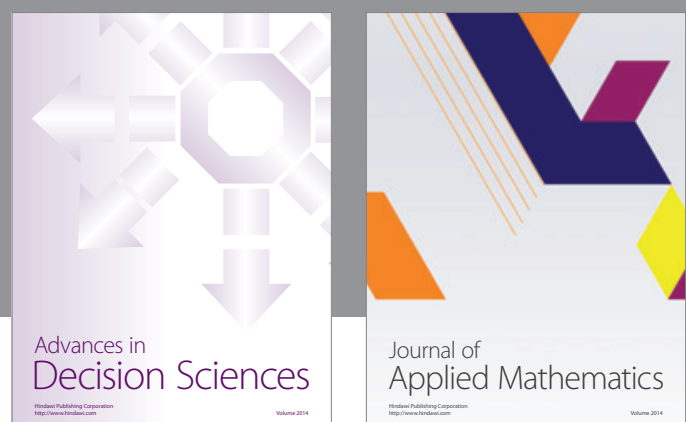

Journal of

Applied Mathematics
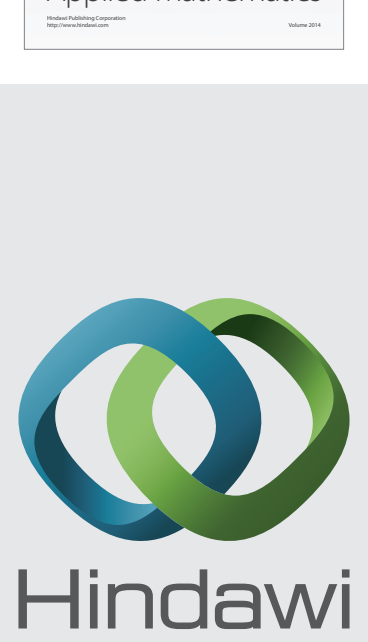

Submit your manuscripts at http://www.hindawi.com
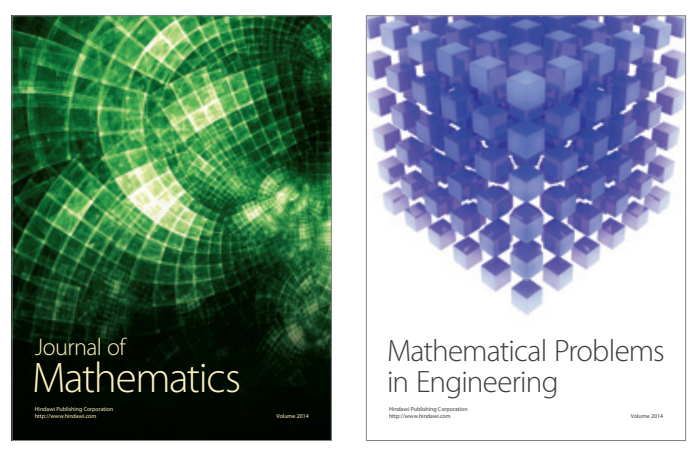

Mathematical Problems in Engineering
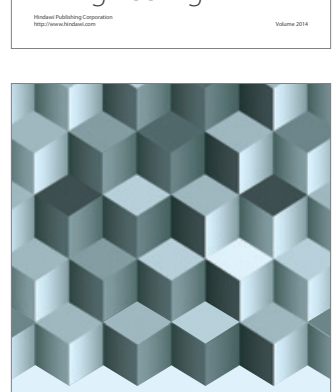

Journal of

Function Spaces
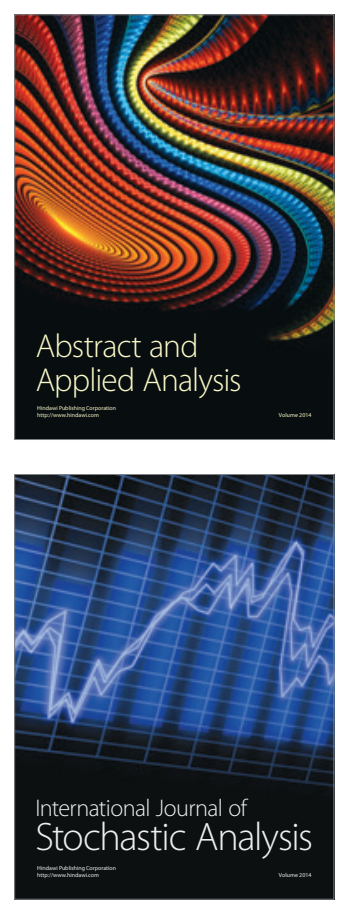

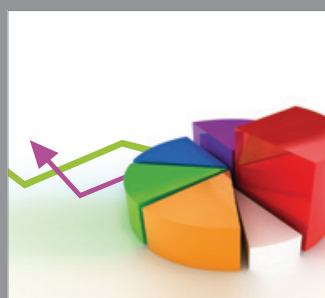

ournal of

Probability and Statistics

Promensencen
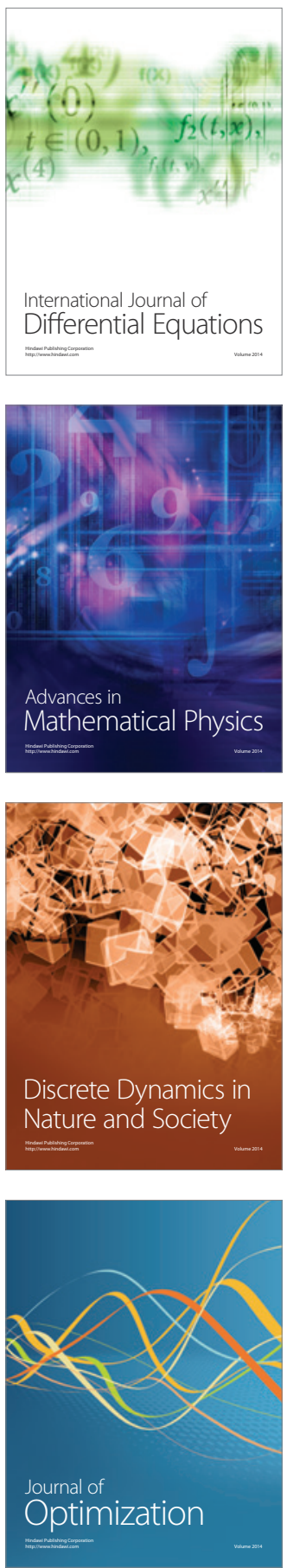